\title{
SEMILOCAL CONVERGENCE OF NEWTON'S METHOD FOR SINGULAR SYSTEMS WITH CONSTANT RANK DERIVATIVES
}

\author{
IOANNIS K. ARgYros ${ }^{a}$ AND SAÏD HILOUT ${ }^{b}$
}

\begin{abstract}
We provide a semilocal convergence result for approximating a solution of a singular system with constant rank derivatives, using Newton's method in an Euclidean space setting. Our approach uses more precise estimates and a combination of two Lipschitz-type conditions leading to the following advantages over earlier works [13], [16], [17], [29]: tighter bounds on the distances involved, and a more precise information on the location of the solution. Numerical examples are also provided in this study.
\end{abstract}

\section{INTRODUCTION}

In this study we are concerned with the problem of approximating a locally unique solution $x^{\star}$ of equation

$$
F^{\prime}(x)^{+} F(x)=0,
$$

where, $F$ is a continuously Fréchet-differentiable operator defined on an open, nonempty, convex subset $\mathcal{D}$ of $\mathcal{X}$ in $\mathcal{Y}$, where $X$ and $\mathcal{Y}$ are an Euclidean spaces $(\operatorname{dim} \mathcal{X}=i ; \operatorname{dim} \mathcal{Y}=j ; i, j \in \mathbb{N})$, and for arbitrary $A \in \mathcal{L}(\mathcal{X}, \mathcal{Y}), A^{+}$denotes a Moore-Penrose generalized inverse of $A$, defined as the unique linear operator in $\mathcal{L}(\mathcal{Y}, \mathcal{X})$ satisfying

$$
A A^{+} A=A, \quad A^{+} A A^{+}=A^{+}, \quad\left(A^{+} A\right)^{T}=A^{+} A, \quad\left(A A^{+}\right)^{T}=A A^{+} .
$$

The field of computational sciences has seen a considerable development in mathematics, engineering sciences, and economic equilibrium theory. For example, dynamic systems are mathematically modeled by difference or differential equations, and their solutions usually represent the states of the systems. For the sake of simplicity, assume that a time-invariant system is driven by the equation $\dot{x}=T(x)$,

Received by the editors September 22, 2010. Revised May 10, 2011. Accepted May 16, 2011. 2000 Mathematics Subject Classification. 65H10, 65G99, 65J15, 47H17, 49M15.

Key words and phrases. Newton's method, Euclidean space, singular system, constant rank derivative, Fréchet derivative, Moore-Penrose matrix. 
for some suitable operator $T$, where $x$ is the state. Then the equilibrium states are determined by solving equation (1.1). Similar equations are used in the case of discrete systems. The unknowns of engineering equations can be functions (difference, differential, and integral equations), vectors (systems of linear or nonlinear algebraic equations), or real or complex numbers (single algebraic equations with single unknowns). Except in special cases, the most commonly used solution methods are iterative-when starting from one or several initial approximations a sequence is constructed that converges to a solution of the equation. Iteration methods are also applied for solving optimization problems. In such cases, the iteration sequences converge to an optimal solution of the problem at hand. Since all of these methods have the same recursive structure, they can be introduced and discussed in a general framework. We note that in computational sciences, the practice of numerical analysis for finding such solutions is essentially connected to variants of Newton's method.

Newton's method (NM)

$$
x_{n+1}=x_{n}-F^{\prime}\left(x_{n}\right)^{+} F\left(x_{n}\right) \quad(n \geq 0), \quad\left(x_{0} \in \mathcal{D}\right),
$$

is undoubtedly the must popular way for generating a sequence $\left\{x_{n}\right\}$ approximating a solution $x^{\star}$. A survey on local as well as semilocal convergence results for Newtontype methods can be found in [1]-[29] and the references there.

In this study, we are motivated by the recent elegant study by $\mathrm{Xu}$ and $\mathrm{Li}$ [29] (which improved earlier results [9]), and optimization considerations. Using a combination of two Lipschitz-type conditions, we provide a new semilocal convergence result for Newton's method (1.3) with the following advantages (under the same hypotheses and computational cost) over [29]:

(a) Tighter error bounds estimates on $\left\|x_{n+1}-x_{n}\right\|$ and $\left\|x_{n}-x^{\star}\right\|(n \geq 0)$;

(b) A more precise information on the location of the solution $x^{\star}$.

Numerical examples and applications are also provided in this study.

It is well known that the solution set of equation (1.1) may contain elements not in the solution set of equation ([4], [8])

$$
F(x)=0 .
$$

The paper is organized as follows: Preliminary results are provided in Section 2. The semilocal convergence of Newton's method is given in Section 3. Finally, the numerical examples and applications are provided in Section 4. 


\section{Preliminary Results}

In order for us to make the study as self contained as possible, we re-introduce some basic notions and results [4], [21], [29]. Let $\mathcal{X}, \mathcal{Y}$ denote the two Euclidean spaces, and $F: \mathcal{D} \subseteq \mathcal{X} \longrightarrow \mathcal{Y}$ as defined in the introduction. We suppose $\operatorname{rank} F^{\prime}(x) \leq r(x \in \mathcal{D})$ and $r \leq \min \{i, j\}$ is a positive integer. Denote by $I_{\mathcal{X}}$ the identity on $\mathcal{X}$ and $U(z, R)$ the open ball with radius $R>0$ and center $z \in \mathcal{X}$. Finally, for matrix $\mathcal{M}$, denote by $\operatorname{Ker} \mathcal{M}$ and $\operatorname{Im} \mathcal{M}$ the kernel and image of $\mathcal{M}$, respectively, and $\Pi_{E}$ the orthogonal projection onto a subspace $E$ of $\mathcal{X}$. We have the following statments:

$$
\mathcal{M}^{+} \mathcal{M}=\Pi_{K e r \mathcal{M}^{\perp}} \quad \text { and } \quad \mathcal{M} \mathcal{M}^{+}=\Pi_{I m \mathcal{M}}
$$

We will use two lemmas of perturbations bounds for Moore-Penrose inverse, whose proofs can be found in [21].

Lemma 2.1. Let $\mathcal{M}$ and $\mathcal{N}$ be two $i \times j$ matrices, with $\operatorname{rank} \mathcal{N}=\operatorname{rank} \mathcal{M}=r$, and $\left\|\mathcal{M}^{+}\right\|\|\mathcal{N}-\mathcal{M}\|<1$. Then, the following holds

$$
\left\|\mathcal{N}^{+}-\mathcal{M}^{+}\right\| \leq c \frac{\left\|\mathcal{M}^{+}\right\|^{2}\|\mathcal{N}-\mathcal{M}\|}{1-\left\|\mathcal{M}^{+}\right\|\|\mathcal{N}-\mathcal{M}\|}
$$

where,

$$
c=\left\{\begin{array}{ccc}
\frac{1+\sqrt{5}}{2} & \text { if } & r<\min \{i, j\} \\
\sqrt{2} & \text { if } & r=\min \{i, j\}(i \neq j) \\
1 & \text { if } & r=i=j .
\end{array}\right.
$$

We only study singular case when $r<\min \{i, j\}$ (i.e., $c=\frac{1+\sqrt{5}}{2}$ in Lemma 2.1). The case $r=\min \{i, j\}$ can also studied similarly.

Lemma 2.2. Let $\mathcal{M}$ and $\mathcal{N}$ be two $i \times j$ matrices, with $\operatorname{rank}(\mathcal{M}+\mathcal{N}) \leq \operatorname{rank} \mathcal{M}=r$, and $\left\|\mathcal{M}^{+}\right\|\|\mathcal{N}\|<1$. Then, the following hold

$$
\operatorname{rank}(\mathcal{M}+\mathcal{N})=r
$$

and

$$
\left\|(\mathcal{M}+\mathcal{N})^{+}\right\| \leq \frac{\left\|\mathcal{M}^{+}\right\|}{1-\left\|\mathcal{M}^{+}\right\|\|\mathcal{N}\|}
$$

We need the following definition.

Definition 2.3. Let $R>0$ be such that $U\left(x_{0}, R\right) \subseteq \mathcal{D}$, and $L_{0}(u), L(u)$ be positive nondecreasing functions on $[0,+\infty)$. 
(a) Mapping $F^{\prime}$ satisfies the center-Lipschitz condition with $L_{0}$-average in $U\left(x_{0}, R\right)$, if, for any $x \in U\left(x_{0}, R\right)$, the following holds

$$
\left\|F\left(x_{0}\right)^{+}\right\|\left\|F^{\prime}(x)-F^{\prime}\left(x_{0}\right)\right\| \leq \int_{0}^{\left\|x-x_{0}\right\|} L_{0}(u) d u .
$$

(b) Mapping $F^{\prime}$ satisfies the center-Lipschitz condition in the inscribed sphere with $L$-average in $U\left(x_{0}, R\right)$, if, for any $x, y \in U\left(x_{0}, R\right)$ with $\left\|x-x_{0}\right\|+\|$ $y-x \|<R$, the following holds

$$
\left\|F\left(x_{0}\right)^{+}\right\|\left\|F^{\prime}(y)-F^{\prime}(x)\right\| \leq \int_{\left\|x-x_{0}\right\|}^{\left\|x-x_{0}\right\|+\|y-x\|} L(u) d u .
$$

Remark 2.4. (i) By Definition 2.3, if $F^{\prime}$ satisfies the center-Lipschitz condition in the inscribed sphere with $L$-average in $U\left(x_{0}, R\right)$, then, $F^{\prime}$ satisfies the centerLipschitz condition with $L$-average in $U\left(x_{0}, R\right)$.

(ii) Note that

$$
L_{0}(u) \leq L(u) \quad u \in[0,+\infty),
$$

and $\frac{L(u)}{L_{0}(u)}$ can be arbitrarily large [1]-[7].

(iii) If equality holds in (2.5), then our Definition 2.3 coincides with [29, Definition 2.2, p. 691].

We need the following results.

Lemma 2.5. Assume that $F^{\prime}$ satisfies the center-Lipschitz condition with $L_{0^{-}}$ average in $U\left(x_{0}, R\right)$, and for $x \in U\left(x_{0}, R\right)$ :

$$
\operatorname{rank} F^{\prime}(x) \leq \operatorname{rank} F^{\prime}\left(x_{0}\right)=r \text { and } \int_{0}^{\left\|x-x_{0}\right\|} L_{0}(u) d u<1 .
$$

Then, for $x \in U\left(x_{0}, R\right)$, the following hold:

$$
\begin{gathered}
\operatorname{rank} F^{\prime}(x)=r \\
\left\|F^{\prime}(x)\right\| \leq\left\|F^{\prime}\left(x_{0}\right)\right\|+\frac{1}{\left\|F^{\prime}\left(x_{0}\right)^{+}\right\|} \int_{0}^{\left\|x-x_{0}\right\|} L_{0}(u) d u,
\end{gathered}
$$

and

$$
\left\|F^{\prime}(x)^{+}\right\| \leq \frac{\left\|F^{\prime}\left(x_{0}\right)^{+}\right\|}{1-\int_{0}^{\left\|x-x_{0}\right\|} L_{0}(u) d u} .
$$


Proof. (a) We have:

$$
\Pi_{K e r F^{\prime}\left(x_{0}\right)}+F^{\prime}\left(x_{0}\right)^{+} F^{\prime}(x)=I_{\mathcal{X}}-F^{\prime}\left(x_{0}\right)^{+}\left(F^{\prime}\left(x_{0}\right)-F^{\prime}(x)\right),
$$

and

$$
\left\|F^{\prime}\left(x_{0}\right)^{+}\right\|\left\|F^{\prime}\left(x_{0}\right)-F^{\prime}(x)\right\| \leq \int_{0}^{\left\|x-x_{0}\right\|} L_{0}(u) d u<1 .
$$

Hence $\Pi_{K e r F^{\prime}\left(x_{0}\right)}+F^{\prime}\left(x_{0}\right)^{+} F^{\prime}(x)$ is nonsingular. Using (2.1), we obtain

$$
\begin{aligned}
\Pi_{I m F^{\prime}\left(x_{0}\right)} F^{\prime}(x) & =F^{\prime}\left(x_{0}\right) F^{\prime}\left(x_{0}\right)^{+} F^{\prime}(x)+F^{\prime}\left(x_{0}\right) \Pi_{K e r F^{\prime}\left(x_{0}\right)} \\
& =F^{\prime}\left(x_{0}\right)\left(F^{\prime}\left(x_{0}\right)^{+} F^{\prime}(x)+\Pi_{K e r} F^{\prime}\left(x_{0}\right)\right) .
\end{aligned}
$$

That is, we get

$$
\operatorname{rank}\left(\Pi_{I m F^{\prime}\left(x_{0}\right)} F^{\prime}(x)\right)=\operatorname{rank} F^{\prime}\left(x_{0}\right)=r,
$$

which together with $\operatorname{rank} F^{\prime}(x) \leq \operatorname{rank} F^{\prime}\left(x_{0}\right)=r$, imply (2.6).

(b) Using (2.3), we get

$$
\begin{aligned}
\left\|F^{\prime}(x)\right\| & \leq\left\|F^{\prime}\left(x_{0}\right)\right\|+\left\|F^{\prime}(x)-F^{\prime}\left(x_{0}\right)\right\| \\
& \leq\left\|F^{\prime}\left(x_{0}\right)\right\|+\frac{1}{\left\|F^{\prime}\left(x_{0}\right)+\right\|} \int_{0}^{\left\|x-x_{0}\right\|} L_{0}(u) d u .
\end{aligned}
$$

(c) Set $\mathcal{M}=F^{\prime}\left(x_{0}\right)$, and $\mathcal{N}=F^{\prime}(x)-F^{\prime}\left(x_{0}\right)$. In view of $(2.10)$, $\operatorname{rank} \mathcal{M}=r$, $\left\|\mathcal{M}^{+}\right\|\|\mathcal{N}\| \leq \int_{0}^{\left\|x-x_{0}\right\|} L_{0}(u) d u<1$. By Lemma 2.2, we get

$$
\left\|F^{\prime}(x)^{+}\right\|=\left\|(\mathcal{M}+\mathcal{N})^{+}\right\| \leq \frac{\left\|\mathcal{M}^{+}\right\|}{1-\left\|\mathcal{M}^{+}\right\|\|\mathcal{N}\|} \leq \frac{\left\|F^{\prime}\left(x_{0}\right)^{+}\right\|}{1-\int_{0}^{\left\|x-x_{0}\right\|} L_{0}(u) d u} .
$$

That completes the proof of Lemma 2.5.

Remark 2.6. If $L_{0}(u)=L(u)$, Lemma 2.5 coincides with [29, Lemma 2.3, p. 692].

Lemma $2.7([29])$. Assume that $F^{\prime}$ satisfies the center-Lipschitz condition in the inscribed sphere with $L$-average in $U\left(x_{0}, R\right)$, and $\int_{0}^{R} L(u) d u<1$. Let $x \in U\left(x_{0}, R\right)$, and $y$ such that $\left\|x-x_{0}\right\|+\|y-x\|<R$, and $\max \left\{\operatorname{rank} F^{\prime}(x), \operatorname{rank} F^{\prime}(y)\right\} \leq$ $\operatorname{rank} F^{\prime}\left(x_{0}\right)=r$. Then

$$
\begin{aligned}
& \left\|F^{\prime}(y)^{+}-F^{\prime}(x)^{+}\right\| \\
& \leq \frac{1+\sqrt{5}}{2} \frac{\left\|F^{\prime}(x)^{+}\right\|^{2}\left\|F^{\prime}\left(x_{0}\right)^{+}\right\|^{-1} \int_{0}^{\|y-x\|} L\left(\left\|x-x_{0}\right\|+u\right) d u}{1-\left\|F^{\prime}(x)^{+}\right\|\left\|F^{\prime}\left(x_{0}\right)^{+}\right\|^{-1} \int_{0}^{\|y-x\|} L\left(\left\|x-x_{0}\right\|+u\right) d u} .
\end{aligned}
$$


Lemma 2.8 ([20]). Let $\varrho \geq 0$, and define functions $\psi_{1}$ and $\psi_{2}$ on $(0,+\infty)$ by:

$$
\psi_{1}(t)=\frac{1}{t} \int_{0}^{t} L(u) d u \quad \text { and } \quad \psi_{2}(t)=\frac{1}{t} \int_{0}^{t}(t-u) L(\varrho+u) d u .
$$

Then $\psi_{1}$ and $\psi_{2}$ are positive nondecreasing on $(0,+\infty)$.

Lemma 2.9 ([27]). Assume that $F^{\prime}$ satisfies the center-Lipschitz condition in the inscribed sphere with $L$-average in $U\left(x_{0}, R\right)$. Let $x, y \in U\left(x_{0}, R\right)$ with $\left\|x-x_{0}\right\|$ $+\|y-x\|<R$. Then, the following hold:

$$
\begin{aligned}
& \left\|F(x)-F(y)-F^{\prime}(x)(x-y)\right\| \\
& \leq \frac{1}{\left\|F^{\prime}\left(x_{0}\right)^{+}\right\|} \int_{0}^{\|y-x\|}(\|y-x\|-u) L\left(\left\|x-x_{0}\right\|+u\right) d u .
\end{aligned}
$$

and

$$
\begin{aligned}
& \|F(y)-F(x)\| \\
& \leq \frac{1}{\left\|F^{\prime}\left(x_{0}\right)^{+}\right\|} \int_{0}^{\|y-x\|}(\|y-x\|-u) L\left(\left\|x-x_{0}\right\|+u\right) d u+\left\|F^{\prime}(x)\right\|\|y-x\| .
\end{aligned}
$$

\section{Semilocal Convergence of Newton's Method}

Definition 3.1. Let

$$
\mathcal{S}=\left\{x: F^{\prime}(x)^{+} F(x)=0\right\} .
$$

Newton's method converges, in general to a point in $\mathcal{S}$ rather than a solution of the equation $F(x)=0$, when $F$ is a singular system.

Definition 3.2. It is convenient for us to define some constants. Let $a \in[0,1)$, $b=\frac{1}{1-a}$,

$$
\begin{array}{cc}
K=\left\|F^{\prime}\left(x_{0}\right)^{+}\right\|\left\|F^{\prime}\left(x_{0}\right)\right\|, & \beta=\left\|F^{\prime}\left(x_{0}\right)^{+}\right\|\left\|F\left(x_{0}\right)\right\|, \\
\delta_{1}=\int_{0}^{\beta} L(b \beta+u) d u, & \overline{\delta_{1}}=\int_{0}^{\beta} L_{0}(b \beta+u) d u, \\
\delta_{2}=\int_{0}^{b \beta} L(u) d u, & \overline{\delta_{2}}=\int_{0}^{b \beta} L_{0}(u) d u, \\
\Delta_{1}=\int_{0}^{\beta}(\beta-u) L(b \beta+u) d u, & \overline{\Delta_{1}}=\int_{0}^{\beta}(\beta-u) L_{0}(b \beta+u) d u, \\
\Delta_{2}=\int_{0}^{b \beta}(b \beta-u) L(b \beta+u) d u, & \overline{\Delta_{2}}=\int_{0}^{b \beta}(b \beta-u) L_{0}(b \beta+u) d u
\end{array}
$$




$$
p=\frac{\Delta_{1}}{\beta\left(1-\overline{\delta_{2}}\right)}+\frac{1+\sqrt{5}}{2} \frac{\delta_{1}\left(\beta+\beta \overline{\delta_{2}}+(b+1) \beta K+\Delta_{1}+\overline{\Delta_{2}}\right)}{\beta\left(1-\overline{\delta_{2}}\right)\left(1-\delta_{1}-\overline{\delta_{2}}\right)},
$$

and

$$
p_{0}=\frac{\overline{\Delta_{1}}}{\beta}+\frac{1+\sqrt{5}}{2} \frac{\overline{\delta_{1}}\left(\beta+\beta K+\overline{\Delta_{1}}\right)}{\beta\left(1-\overline{\delta_{1}}\right)} .
$$

We shall provide a semilocal convergence analysis for Newton's method.

Theorem 3.3. Let $F: \mathcal{D} \subseteq \mathcal{X} \longrightarrow \mathcal{Y}$ satisfying (2.3), (2.4) in $\mathcal{D}_{0}=U\left(x_{0}, b \beta\right)$, and $\operatorname{rank} F^{\prime}(x) \leq \operatorname{rank} F^{\prime}\left(x_{0}\right)$ far all $x \in \mathcal{D}_{0}$. Assume:

$$
\delta_{1}+\overline{\delta_{2}}<1 \text { and } p \leq a .
$$

Then, sequence $\left\{x_{n}\right\}$ generated by Newton's method is well defined, and converges to a point $x^{\star} \in \mathcal{S}$. Moreover, the following estimates hold:

$$
\begin{gathered}
\left\|x_{2}-x_{1}\right\| \leq p_{0} \quad\left\|x_{1}-x_{0}\right\| \leq p \quad\left\|x_{1}-x_{0}\right\|, \\
\left\|x_{n+1}-x_{n}\right\| \leq a^{n} \quad\left\|x_{1}-x_{0}\right\|, \quad(n \geq 2)
\end{gathered}
$$

and

$$
\left\|x_{0}-x^{\star}\right\| \leq b \quad\left\|x_{1}-x_{0}\right\| .
$$

Proof. We shall show (3.3), and

$$
\left\|x_{k+1}-x_{k}\right\| \leq a \quad\left\|x_{k}-x_{k-1}\right\|, \quad k \geq 2
$$

by induction on $k$.

For $x \in \mathcal{D}_{0}$, we have

$$
\int_{0}^{\left\|x-x_{0}\right\|} L_{0}(u) d u \leq \int_{0}^{b \beta} L_{0}(u) d u=\overline{\delta_{2}}<1 .
$$

Using (2.6), we get $\operatorname{rank} F^{\prime}(x) \leq \operatorname{rank} F^{\prime}\left(x_{0}\right)$ for $x \in \mathcal{D}_{0}$. We also have:

$$
x_{1}-x_{0}=-F^{\prime}\left(x_{0}\right)^{+} F\left(x_{0}\right) \in \operatorname{Ker} F^{\prime}\left(x_{0}\right)^{\perp} \text { and } F^{\prime}\left(x_{0}\right)^{+} F^{\prime}\left(x_{0}\right)=\Pi_{\operatorname{Ker} F^{\prime}\left(x_{0}\right)^{\perp}},
$$

so,

$$
\begin{aligned}
x_{2}-x_{1}= & F^{\prime}\left(x_{0}\right)^{+} F^{\prime}\left(x_{0}\right)\left(x_{1}-x_{0}\right)+F^{\prime}\left(x_{0}\right)^{+} F\left(x_{0}\right)-F^{\prime}\left(x_{1}\right)^{+} F\left(x_{1}\right) \\
= & F^{\prime}\left(x_{0}\right)^{+}\left(F^{\prime}\left(x_{0}\right)\left(x_{1}-x_{0}\right)+F\left(x_{0}\right)-F\left(x_{1}\right)\right) \\
& +\left(F^{\prime}\left(x_{0}\right)^{+}-F^{\prime}\left(x_{1}\right)^{+}\right)\left(F\left(x_{1}\right)-F\left(x_{0}\right)\right)+\left(F^{\prime}\left(x_{0}\right)^{+}-F^{\prime}\left(x_{1}\right)^{+}\right) F\left(x_{0}\right) .
\end{aligned}
$$

Since $\left\|x_{1}-x_{0}\right\| \leq \beta$, and $L_{0}(u)$ is nondecreasing, we deduce from Lemmas 2.6-2.8 that 


$$
\begin{aligned}
& \left\|x_{2}-x_{1}\right\| \\
& \leq \int_{0}^{\left\|x_{1}-x_{0}\right\|}\left(\left\|x_{1}-x_{0}\right\|-u\right) L_{0}\left(\left\|x_{1}-x_{0}\right\|+u\right) d u \\
& +\frac{1+\sqrt{5}}{2} \frac{\int_{0}^{\left\|x_{1}-x_{0}\right\|} L_{0}(u) d u}{1-\int_{0}^{\left\|x_{1}-x_{0}\right\|}} L_{0}(u) d u \\
& \quad+K \| x_{0}^{\left\|x_{1}-x_{0}\right\|}\left(\left\|x_{1}-x_{0}\right\|-u\right) L_{0}\left(\left\|x_{1}-x_{0}\right\|+u\right) d u \\
& \leq\left(\frac{\overline{\Delta_{1}}}{\beta}+\frac{1+\sqrt{5}}{2} \frac{\overline{\delta_{1}}}{1-\overline{\delta_{1}}}\left(\frac{\overline{\Delta_{1}}}{\beta}+K+\frac{1+\sqrt{5}}{2} \frac{\int_{0}^{\left\|x_{1}-x_{0}\right\|} L_{0}(u) d u}{1-\int_{0}^{\left\|x_{1}-x_{0}\right\|} L_{0}(u) d u}\right.\right. \\
& =\left(\frac{\overline{\Delta_{1}}}{\beta}+\frac{1+\sqrt{5}}{2} \frac{\overline{\delta_{1}}}{\beta\left(1-\overline{\delta_{1}}\right)}\left(\beta+\beta K+\overline{\Delta_{1}}\right)\right)\left\|x_{1}-x_{0}\right\| \\
& \leq p_{0}\left\|x_{1}-x_{0}\right\| \leq a\left\|x_{1}-x_{0}\right\|, \\
& \text { which implies }(3.4), \text { and }(3.5) \text { for } k=1 .
\end{aligned}
$$

Assume that (3.6) holds for $k=2, \cdots, k$. Using (3.8) by replacing $x_{2}, x_{1}$ by $x_{n+1}$ and $x_{n}$ respectively, we get

$$
\begin{aligned}
\left\|x_{n+1}-x_{n}\right\| \leq & \left\|F^{\prime}\left(x_{n-1}\right)^{+}\right\|\left\|F^{\prime}\left(x_{n-1}\right)\left(x_{n}-x_{n-1}\right)+F\left(x_{n-1}\right)-F\left(x_{n}\right)\right\| \\
& +\left\|F^{\prime}\left(x_{n-1}\right)^{+}-F^{\prime}\left(x_{n}\right)^{+}\right\|\left\|F\left(x_{n}\right)-F\left(x_{n-1}\right)\right\| \\
& +\left\|F^{\prime}\left(x_{n-1}\right)^{+}-F^{\prime}\left(x_{n}\right)^{+}\right\|\left\|F\left(x_{n-1}\right)\right\| \\
& =\mathcal{B}_{1}+\mathcal{B}_{2}+\mathcal{B}_{3} .
\end{aligned}
$$

We shall estimate $\mathcal{B}_{1}, \mathcal{B}_{2}$, and $\mathcal{B}_{3}$. By induction hypothesis (3.6), we have

$$
\left\|x_{i}-x_{0}\right\| \leq \sum_{k=1}^{n}\left\|x_{k}-x_{k-1}\right\| \leq b\left\|x_{1}-x_{0}\right\| \leq b \beta \quad \text { for } i=n, n-1 .
$$

Thus, (2.8) implies

$$
\left\|F^{\prime}\left(x_{n-1}\right)^{+}\right\| \leq \frac{\left\|F^{\prime}\left(x_{0}\right)^{+}\right\|}{1-\int_{0}^{\left\|x_{n-1}-x_{0}\right\|} L_{0}(u) d u} \leq \frac{\left\|F^{\prime}\left(x_{0}\right)^{+}\right\|}{1-\int_{0}^{b \beta} L_{0}(u) d u}=\frac{\left\|F^{\prime}\left(x_{0}\right)^{+}\right\|}{1-\overline{\delta_{2}}} .
$$

Using (3.11), we have

$$
L_{0}\left(\left\|x_{n}-x_{0}\right\|+u\right) \leq L_{0}(b \beta+u), \quad \forall u \geq 0 .
$$


By induction hypothesis, (3.13), and Lemma 2.8, we obtain

$$
\begin{aligned}
& \int_{0}^{\left\|x_{n}-x_{n-1}\right\|}\left(\left\|x_{n}-x_{n-1}\right\|-u\right) L\left(\left\|x_{n}-x_{0}\right\|+u\right) d u \\
& \leq \frac{1}{\left\|x_{n}-x_{n-1}\right\|} \int_{0}^{\left\|x_{n}-x_{n-1}\right\|}\left(\left\|x_{n}-x_{n-1}\right\|-u\right) L(b \beta+u) d u\left\|x_{n}-x_{n-1}\right\| \\
& \leq \frac{\Delta_{1}}{\beta}\left\|x_{n}-x_{n-1}\right\| .
\end{aligned}
$$

By (2.13), we deduce the following estimate of $\mathcal{B}_{1}$ :

$$
\begin{aligned}
\mathcal{B}_{1} & \leq \frac{\left\|F^{\prime}\left(x_{n-1}\right)^{+}\right\|}{\left\|F^{\prime}\left(x_{0}\right)^{+}\right\|} \int_{0}^{\left\|x_{n}-x_{n-1}\right\|}\left(\left\|x_{n}-x_{n-1}\right\|-u\right) L\left(\left\|x_{n}-x_{0}\right\|+u\right) d u \\
& \leq \frac{\Delta_{1}}{\beta\left(1-\overline{\delta_{2}}\right)}\left\|x_{n}-x_{n-1}\right\|
\end{aligned}
$$

We shall now estimate $\mathcal{B}_{2}$ and $\mathcal{B}_{3}$. By induction hypothesis, (3.13), and Lemma 2.8, we have:

$$
\begin{aligned}
& \int_{0}^{\left\|x_{n}-x_{n-1}\right\|} L\left(\left\|x_{n-1}-x_{0}\right\|+u\right) d u \\
& \leq \frac{1}{\left\|x_{1}-x_{0}\right\|} \int_{0}^{\left\|x_{1}-x_{0}\right\|} L(b \beta+u) d u\left\|x_{n}-x_{n-1}\right\| \\
& \leq \frac{\delta_{1}}{\beta}\left\|x_{n}-x_{n-1}\right\| .
\end{aligned}
$$

From Lemma 2.7, we have:

$$
\begin{aligned}
& \left\|F^{\prime}\left(x_{n}\right)^{+}-F^{\prime}\left(x_{n-1}\right)^{+}\right\| \\
& \leq \frac{1+\sqrt{5}}{2} \frac{\left\|F^{\prime}\left(x_{n-1}\right)^{+}\right\|^{2}\left\|F^{\prime}\left(x_{0}\right)^{+}\right\|^{-1} \int_{0}^{\left\|x_{n}-x_{n-1}\right\|} L\left(\left\|x_{n-1}-x_{0}\right\|+u\right) d u}{1-\left\|F^{\prime}\left(x_{n-1}\right)^{+}\right\|\left\|F^{\prime}\left(x_{0}\right)^{+}\right\|^{-1} \int_{0}^{\left\|x_{n}-x_{n-1}\right\|} L\left(\left\|x_{n-1}-x_{0}\right\|+u\right) d u} \\
& \leq \frac{1+\sqrt{5}}{2} \frac{\left\|F^{\prime}\left(x_{0}\right)^{+}\right\|}{\left(1-\overline{\delta_{2}}\right)^{2}} \frac{\delta_{1}}{\beta} \frac{\left\|x_{n}-x_{n-1}\right\|}{1-\frac{\delta_{1}}{\beta\left(1-\overline{\delta_{2}}\right)}\left\|x_{n}-x_{n-1}\right\|} \\
& \leq \frac{1+\sqrt{5}}{2} \frac{\left\|F^{\prime}\left(x_{0}\right)^{+}\right\| \delta_{1}}{\beta\left(1-\overline{\delta_{2}}\right)\left(1-\delta_{1}-\overline{\delta_{2}}\right)}\left\|x_{n}-x_{n-1}\right\| .
\end{aligned}
$$

We also have: 


$$
\begin{aligned}
\| & F\left(x_{n}\right)-F\left(x_{n-1}\right) \| \\
\leq & \frac{1}{\left\|F^{\prime}\left(x_{0}\right)^{+}\right\|} \int_{0}^{\left\|x_{n}-x_{n-1}\right\|}\left(\left\|x_{n}-x_{n-1}\right\|-u\right) L\left(\left\|x_{n-1}-x_{0}\right\|+u\right) d u \\
& +\left\|F^{\prime}\left(x_{n-1}\right)\right\|\left\|x_{n}-x_{n-1}\right\| \\
\leq & \frac{\Delta_{1}}{\left\|F^{\prime}\left(x_{0}\right)^{+}\right\|}\left(K+\int_{0}^{\left\|x_{n-1}-x_{0}\right\|} L_{0}(u) d u\right) \beta \\
\leq & \frac{\beta \overline{\delta_{2}}+\beta K+\Delta_{1}}{\left\|F^{\prime}\left(x_{0}\right)^{+}\right\|} .
\end{aligned}
$$

By (3.15), and (3.16), we obtain the estimate of $\mathcal{B}_{2}$ :

$$
\begin{aligned}
\mathcal{B}_{2} & \leq \frac{1+\sqrt{5}}{2} \frac{\left\|F^{\prime}\left(x_{0}\right)^{+}\right\| \delta_{1}}{\beta\left(1-\overline{\delta_{2}}\right)\left(1-\delta_{1}-\overline{\delta_{2}}\right)}\left\|x_{n}-x_{n-1}\right\| \frac{\beta \overline{\delta_{2}}+\beta K+\Delta_{1}}{\left\|F^{\prime}\left(x_{0}\right)^{+}\right\|} \\
& =\frac{1+\sqrt{5}}{2} \frac{\delta_{1}\left(\beta \overline{\delta_{2}}+\beta K+\Delta_{1}\right)}{\beta\left(1-\overline{\delta_{2}}\right)\left(1-\delta_{1}-\overline{\delta_{2}}\right)}\left\|x_{n}-x_{n-1}\right\| .
\end{aligned}
$$

For estimating $\mathcal{B}_{3}$, we first estimate $\left\|F\left(x_{n-1}\right)\right\|$. By (2.13), we have

$$
\begin{aligned}
& \left\|F\left(x_{n-1}\right)\right\| \\
& \leq\left\|F\left(x_{0}\right)\right\|+\left\|F^{\prime}\left(x_{0}\right)\right\|\left\|x_{n-1}-x_{0}\right\|+\left\|F\left(x_{n-1}\right)-F\left(x_{0}\right)-F^{\prime}\left(x_{0}\right)\left(x_{n-1}-x_{0}\right)\right\| \\
& \leq\left\|F\left(x_{0}\right)\right\|+b \beta\left\|F^{\prime}\left(x_{0}\right)\right\|+\frac{1}{\left\|F^{\prime}\left(x_{0}\right)^{+}\right\|} \int_{0}^{\left\|x_{n-1}-x_{0}\right\|}\left(\left\|x_{n-1}-x_{0}\right\|-u\right) L_{0}(u) d u \\
& \leq\left\|F\left(x_{0}\right)\right\|+b \beta\left\|F^{\prime}\left(x_{0}\right)\right\|+\frac{\frac{\Delta_{2}}{\left\|F^{\prime}\left(x_{0}\right)^{+}\right\|} .}{}
\end{aligned}
$$

Hence, by (3.15), and (3.18), we obtain estimate for $\mathcal{B}_{3}$ :

$$
\begin{aligned}
\mathcal{B}_{3} \leq & \frac{1+\sqrt{5}}{2} \frac{\left\|F^{\prime}\left(x_{0}\right)^{+}\right\| \delta_{1}}{\beta\left(1-\overline{\delta_{2}}\right)\left(1-\delta_{1}-\overline{\delta_{2}}\right)}\left\|x_{n}-x_{n-1}\right\|\left(\left\|F\left(x_{0}\right)\right\|\right. \\
& \left.+b \beta\left\|F^{\prime}\left(x_{0}\right)^{+}\right\|+\frac{\overline{\Delta_{2}}}{\left\|F^{\prime}\left(x_{0}\right)^{+}\right\|}\right) \\
\leq & \frac{1+\sqrt{5}}{2} \frac{\delta_{1}\left(\beta+b \beta K+\overline{\Delta_{2}}\right)}{\beta\left(1-\overline{\delta_{2}}\right)\left(1-\delta_{1}-\overline{\delta_{2}}\right)}\left\|x_{n}-x_{n-1}\right\| .
\end{aligned}
$$

Using (3.14), (3.17), and (3.19), we conclude

$$
\begin{aligned}
\left\|x_{n+1}-x_{n}\right\| & \leq \mathcal{B}_{1}+\mathcal{B}_{2}+\mathcal{B}_{3} \\
& \leq p\left\|x_{n}-x_{n-1}\right\| \leq a\left\|x_{n}-x_{n-1}\right\|,
\end{aligned}
$$

and the induction is completed. 
By (3.4), $\left\{x_{n}\right\}$ is a complete sequence, and as such that it converges to $x^{\star}$ in $\mathcal{X}$. Letting $n \longrightarrow \infty$ in (1.3), we get $F^{\prime}\left(x^{\star}\right)^{+} F\left(x^{\star}\right)=0$, and $x^{\star} \in \mathcal{S}$.

Finally, letting $n \longrightarrow \infty$ in

$$
\left\|x_{n+1}-x_{0}\right\| \leq \sum_{i=1}^{n+1}\left\|x_{i}-x_{i-1}\right\| \leq\left(\sum_{i=1}^{n+1} a^{i-1}\right)\left\|x_{1}-x_{0}\right\| \leq b\left\|x_{1}-x_{0}\right\|,
$$

we deduce estimate (3.5).

That completes the proof of Theorem 3.3.

Remark 3.4. In view of the proof of Theorem 3.3, $\delta_{2}, \Delta_{2}$ can replace $\overline{\delta_{2}}$, and $\overline{\Delta_{2}}$, respectively.

\section{Special Cases and Applications}

Let us compare our results with the corresponding in [29].

Case $a=\frac{1}{2}$.

(a) If $L_{0}(u)=L(u)(u \in[0,+\infty))$, then, our Theorem 3.3 is reduces to [29, Theorem 3.1, p. 695].

(b) If

$$
L_{0}(u)<L(u) \quad(u \in[0,+\infty)),
$$

then, our condition (3.2) is weaker, and the error bounds tighter than the corresponding ones in [29]. Indeed, let us define

$$
\begin{gathered}
d_{1}=\int_{0}^{\beta} L(2 \beta+u) d u, \quad d_{2}=\int_{0}^{2 \beta} L(u) d u \\
d_{3}=\int_{0}^{\beta}(\beta-u) L(2 \beta+u) d u, \quad d_{4}=\int_{0}^{2 \beta}(2 \beta-u) L(2 \beta+u) d u,
\end{gathered}
$$

and

$$
d=\frac{d_{3}}{\beta\left(1-d_{2}\right)}+\frac{1+\sqrt{5}}{2} \frac{d_{1}\left(\beta+\beta d_{2}+3 \beta K+d_{3}+d_{4}\right)}{\beta\left(1-d_{2}\right)\left(1-d_{1}-d_{2}\right)} .
$$

The conditions in $[29$, Theorem 3.1, p. 695] are

$$
p_{1}+p_{2}<1 \quad \text { and } \quad d \leq \frac{1}{2} .
$$

But, in view of (4.1), we have:

$$
\delta_{1}<d_{1}, \quad \overline{\delta_{2}}<d_{2}, \quad \overline{\Delta_{1}}<\Delta_{1}<d_{3}, \quad \overline{\Delta_{2}}<\Delta_{2}<d_{4},
$$


SO,

$$
p<d
$$

These advantages are obtained under the same computational cost as in [29], since in practice the computation of function $L$ requires that of $L_{0}$.

Case $a \neq \frac{1}{2}$. This case has not been examined in [29].

(a) If $\frac{1}{2}<a<1$, then $b>2$. This case allows larger range of choices for $\delta_{1}, \overline{\delta_{2}}$, $\Delta_{1}$, and $\overline{\Delta_{2}}$.

(b) If $0 \leq a<\frac{1}{2}$, we obtain $b<2$, and a smaller ratio of convergence. Moreover, in this case, the solution $x^{\star}$ is in $\mathcal{D}_{0} \subseteq U\left(x_{0}, 2 \beta\right)$.

Application 4.1. Let $L_{0}(u)=L_{0}$, and $L(u)=L$ for all $u \in[0, \infty)$, where $L_{0}$ and $L$ are constants, with $0<L_{0} \leq L$. Then, using Definition 3.2, we obtain

$$
\begin{gathered}
\delta_{1}=L \beta, \quad \overline{\delta_{1}}=L_{0} \beta, \quad \delta_{2}=b L \beta, \quad \overline{\delta_{2}}=b L_{0} \beta \\
\Delta_{1}=\frac{L}{2} \beta^{2}, \quad \overline{\Delta_{1}}=\frac{L_{0}}{2} \beta^{2}, \quad \Delta_{2}=b L \beta^{2}, \quad \text { and } \quad \overline{\Delta_{2}}=b L_{0} \beta^{2} .
\end{gathered}
$$

Then, Theorem 3.3 reduces to a Kantorovich-type result.

Let us provide a numerical example, where $L_{0}=L, a<\frac{1}{2}$, and $b<2$.

Example 4.2. Let $\mathcal{X}=\mathcal{Y}=\mathbb{R}^{2}$, be equipped with the $\ell_{1}$-norm. Define $F: \mathcal{X} \longrightarrow$ $\mathcal{Y}$ by:

$$
F(x)=\left(\theta_{1}-\theta_{2}, .5\left(\theta_{1}-\theta_{2}\right)^{2}\right)^{T}, \quad x=\left(\theta_{1}, \theta_{2}\right)^{T} .
$$

$F$ is $\mathcal{C}^{1}$ on $\mathcal{X}$, and for all $x=\left(\theta_{1}, \theta_{2}\right)^{T} \in \mathcal{X}$, we have:

$$
F^{\prime}(x)=\left(\begin{array}{cc}
1 & -1 \\
\theta_{1}-\theta_{2} & \theta_{2}-\theta_{1}
\end{array}\right) \text {. }
$$

Hence, $\operatorname{rank} F^{\prime}(x)=1$ for all $x \in \mathcal{X}$. The Moore-Penrose inverse of $F^{\prime}(x)$ is

$$
F^{\prime}(x)^{+}=\frac{1}{2\left(1+\left(\theta_{1}-\theta_{2}\right)^{2}\right)}\left(\begin{array}{cc}
1 & \theta_{1}-\theta_{2} \\
-1 & \theta_{2}-\theta_{1}
\end{array}\right) .
$$

Let $x_{0}=(.54, .5)^{T}$, and

$$
a=.49, \quad b=1.9607843, \quad\left\|F^{\prime}\left(x_{0}\right)\right\|=1.04, \quad \text { and } \quad\left\|F\left(x_{0}\right)\right\|=.0408 .
$$

Then,

$$
\left\|F^{\prime}\left(x_{0}\right)^{+}\right\|=.99840256 \text {. }
$$


We have also for $x=\left(\theta_{1}, \theta_{2}\right)^{T} \in \mathcal{X}$, and $y=\left(\xi_{1}, \xi_{2}\right)^{T} \in \mathcal{X}$

$\left\|F^{\prime}\left(x_{0}\right)^{+}\right\|\left\|F^{\prime}(x)-F^{\prime}(y)\right\| \leq .99840256\left(\left|\theta_{1}-\xi_{1}\right|+\left|\theta_{2}-\xi_{2}\right|\right) \leq .99840256\|x-y\|$.

We get

$$
\begin{gathered}
L=L_{0}=.99840256, \quad \delta_{1}=.040669753 \\
K=1.038338658, \quad \delta_{2}=.079744613 \\
\beta=.040734824, \quad \Delta_{1}=.000828338, \quad \Delta_{2}=.003248383 \\
\delta_{1}+\delta_{2}=.120414366<1,
\end{gathered}
$$

and

$$
p=.367942966<a=.49 .
$$

Hence, the conclusion of Theorem 3.3 hold. That is Newton's method converges to a point $x^{\star} \in \overline{\mathcal{D}_{0}}$, satisfying $F^{\prime}\left(x^{\star}\right) F\left(x^{\star}\right)=0$, with ratio $a$. The same example in [29] showed that $x^{\star} \in \bar{U}\left(x_{0}, 2 \beta\right) \supseteq \overline{\mathcal{D}_{0}}$, with ration of convergence $a<.5$.

Application 4.3. Theorem 3.3 reduces to a Smale-type result if we define functions $L_{0}, L$ on $\left[0, \frac{1}{\gamma_{0}}\right)$, and $\left[0, \frac{1}{\gamma}\right)$, respectively, by

$$
L_{0}(u)=\frac{2 \gamma_{0}}{\left(1-\gamma_{0} u\right)^{3}}
$$

and

$$
L(u)=\frac{2 \gamma}{(1-\gamma u)^{3}}
$$

If $\gamma_{0}<\gamma$, then, our Theorem 3.3 improves the corresponding results in [29, Section 4]. We leave the details to the motivated reader.

Finally, note that examples where $L_{0}<L$ can be found in [4], in the more general setting of a Banach space.

\section{Conclusion}

Using a combination of two center-Lipschitz-type conditions with average, we provided a semilocal convergence analysis for Newton's method to approximate a locally unique solution of a singular system of equations with constant rank derivatives in Euclidean space setting. Numerical examples and applications further validating the results are also provided in this study. 


\section{REFERENCES}

1. Argyros, I.K.: On the Newton-Kantorovich hypothesis for solving equations. J. Comput. Appl. Math. 169 (2004), 315-332.

2. _ : A unifying local-semilocal convergence analysis and applications for two-point Newton-like methods in Banach space. J. Math. Anal. Appl. 298 (2004), 374-397.

3. _ : On the semilocal convergence of the Gauss-Newton method. Adv. Nonlinear Var. Inequal. 8 (2005), 93-99.

4. _ Computational theory of iterative methods. Studies in Computational Mathematics. Editors: C.K. Chui and L. Wuytack, 15, Elsevier, 2007, New York, U.S.A.

5. _ $\quad$ : On a class of Newton-like methods for solving nonlinear equations. J. Comput. Appl. Math. 228 (2009), 115-122.

6. Argyros, I.K. \& Hilout, S.: Efficient methods for solving equations and variational inequalities. Polimetrica Publisher, Milano, Italy, 2009.

7. __ : Enclosing roots of polynomial equations and their applications to iterative processes. Surveys Math. Appl. 4 (2009), 119-132.

8. Ben-Israel, A. \& Greville, T.N.E.: Generalized inverses. Theory and applications. Second edition. CMS Books in Mathematics/Ouvrages de Mathématiques de la SMC, 15, Springer-Verlag, New York, 2003.

9. Dedieu, J.P. \& Kim, M-H.: Newton's method for analytic systems of equations with constant rank derivatives. J. Complexity 18 (2002), 187-209.

10. Dedieu, J.P. \& Shub, M.: Newton's method for overdetermined systems of equations. Math. Comp. 69 (2000), 1099-1115.

11. Gutiérrez, J.M.: A new semilocal convergence theorem for Newton's method. J. Comput. Appl. Math. 79 (1997), 131-145.

12. Gutiérrez, J.M. \& Hernández, M.A.: Newton's method under weak Kantorovich conditions. IMA J. Numer. Anal. 20 (2000), 521-532.

13. Häubler, W.M.: A Kantorovich-type convergence analysis for the Gauss-Newtonmethod, Numer. Math. 48 (1986), 119-125.

14. Hernández, M.A.: The Newton method for operators with Hölder continuous first derivative. J. Optim. Theory Appl. 109 (2001), 631-648.

15. He, J.S., Wang, J.H. \& Li, C.: Newton's method for underdetermined systems of equations under the $\gamma$-condition. Numer. Funct. Anal. Optim. 28 (2007), 663-679.

16. Hu, N., Shen, W. \& Li, C.: Kantorovich's type theorems for systems of equations with constant rank derivatives. J. Comput. Appl. Math. 219 (2008), 110-122.

17. Kantorovich, L.V. \& Akilov, G.P.: Functional Analysis. Pergamon Press, Oxford, 1982.

18. Kim, M.H.: Computational complexity of the Euler type algorithms for the roots of polynomials. PhD Thesis, CUNY, 1986. 
19. Li, C., Hu, N. \& Wang, J.: Convergence bahavior of Gauss-Newton's method and extensions to the Smale point estimate theory. J. Complexity 26 (2010), 268-295.

20. Li, C., Zhang, W-H. \& Jin, X-Q.: Convergence and uniqueness properties of GaussNewton's method, Comput. Math. Appl. 47 (2004), 1057-1067.

21. Nashed, M.Z. \& Chen, X.: Convergence of Newton-like methods for singular operator equations using outer inverses. Numer. Math. 66 (1993), 235-257.

22. Ortega, L.M. \& Rheinboldt, W.C., Iterative Solution of Nonlinear Equations in Several Variables. Academic press, New York, 1970.

23. Ostrowski, A.M.: Solution of Equations in Euclidean and Banach Spaces. Academic press, New York, 1973.

24. Shub, M. \& Smale, S.: Complexity of Bezout's theorem. IV. Probability of success, extensions. SIAM J. Numer. Anal. 33 (1996), 128-148.

25. Smale, S.: Newton's method estimates from data at one point. The merging of disciplines: new directions in pure, applied, and computational mathematics (Laramie, Wyo., 1985), 185-196, Springer, New York, 1986.

26. Stewart, G.W. \& Sun, J.G.: Matrix perturbation theory. Computer Science and Scientific Computing, Academic Press, Inc., Boston, MA, 1990.

27. Wang, X.H.: Convergence of Newton's method and inverse function theorem in Banach space. Math. Comp. 68 (1999), no. 255, 169-186.

28. Xu, X. \& Li, C.: Convergence of Newton's method for systems of equations with constant rank derivatives. J. Comput. Math. 25 (2007), 705-718.

29. $\quad$ : Convergence criterion of Newton's method for singular systems with constant rank derivatives. J. Math. Anal. Appl. 345 (2008), 689-701.

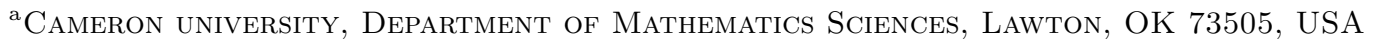

Email address: iargyros@cameron.edu

${ }^{\text {b} P o i t i e r s ~ u n i v e r s i t y, ~ L a b o r a t o i r e ~ d e ~ M a t h e ́ m a t i q u e s ~ e t ~ A p p l i c a t i o n s, ~ B d . ~ P i e r r e ~ e t ~}$ Marie Curie, Téléport 2, B.P. 30179, 86962 Futuroscope Chasseneull Cedex, France

Email address: said.hilout@math.univ-poitiers.fr 